\title{
Botulinum toxin type A in post-stroke lower limb spasticity: a multicenter, double-blind, placebo-controlled trial
}

\author{
Ryuji Kaji $\cdot$ Yuka Osako $\cdot$ Kazuaki Suyama $\cdot$ \\ Toshio Maeda $\cdot$ Yasuyuki Uechi $\cdot$ Masaru Iwasaki
}

Received: 14 October 2009/Revised: 16 February 2010/Accepted: 5 March 2010/Published online: 1 April 2010

(C) The Author(s) 2010. This article is published with open access at Springerlink.com

\begin{abstract}
Lower limb spasticity in post-stroke patients can impair ambulation and reduces activities of daily living (ADL) performance of patients. Botulinum toxin type A (BoNTA) has been shown effective for upper limb spasticity. This study assesses the treatment of lower limb spasticity in a large placebo-controlled clinical trial. In this multicenter, randomized, double-blind, parallel-group, placebo-controlled study, we evaluate the efficacy and safety of one-time injections of botulinum toxin type A (BoNTA) in Japanese patients with post-stroke lower limb spasticity. One hundred twenty patients with lower limb spasticity were randomized to a single treatment with BoNTA $300 \mathrm{U}$ or placebo. The tone of the ankle flexor was assessed at baseline and through 12 weeks using the Modified Ashworth Scale (MAS). Gait pattern and speed of gait were also assessed. The primary endpoint was area under the curve (AUC) of the change from baseline in the MAS ankle score. Significant improvement in spasticity with BoNTA $300 \mathrm{U}$ was demonstrated by a mean difference in the AUC of the change from baseline in the MAS ankle score between the BoNTA and placebo groups $(-3.428 ; 95 \%$ CIs, -5.841 to $-1.016 ; p=0.006 ; t$ test $)$. A significantly greater decrease from baseline in the MAS
\end{abstract}

On behalf of GSK1358820 Spasticity Study Group.

\section{R. Kaji $(\bowtie)$}

Department of Clinical Neuroscience, Institute of Health Biosciences, Tokushima University Graduate School, 2-50-1 Kuramotocho, Tokushima, Tokushima 770-8503, Japan e-mail: rkaji@clin.med.tokushima-u.ac.jp

Y. Osako - K. Suyama - T. Maeda · Y. Uechi · M. Iwasaki Japan Development and Medical Affairs Division,

GlaxoSmithKline K.K., 4-6-15 Sendagaya, Shibuya-ku,

Tokyo 151-8566, Japan ankle score was noted at weeks 4, 6 and 8 in the BoNTA group compared to the placebo group $(p<0.001)$. Significant improvement in the Clinicians Global Impression was noted by the investigator at weeks 4,6 and 8 ( $p=0.016-0.048$, Wilcoxon test), but not by the patient or physical/occupational therapist. Assessments of gait pattern using the Physician's Rating Scale and speed of gait revealed no significant treatment differences but showed a tendency towards improvement with BoNTA. No marked difference was noted in the frequency of treatment-related adverse events between BoNTA and placebo groups. This was the first large-scale trial to indicate that BoNTA significantly reduced spasticity in lower limb muscles.

Keywords Botulinum toxin .

Clinical trials randomized controlled - Spasticity .

Modified Ashworth Scale · Lower limb

\section{Introduction}

Spasticity is defined by Lance as a motor disorder characterized by a velocity-dependent increase in tonic stretch reflexes (muscle tone) with exaggerated tendon jerks, resulting from hyperexcitability of the stretch reflex, as one of the components of upper motor neuron syndrome [1].

BoNTA (botulinum toxin type $\mathrm{A}$, onabotulinumtoxin $\mathrm{A}^{1}$ ) is a specific formulation of a locally injected muscle relaxant whose active ingredient is botulinum toxin type $\mathrm{A}$ produced by Clostridium botulinum. Botulinum toxin type

\footnotetext{
1 In 2009, the American Medical Association United States Adopted Names Council (USAN) published "onabotulinumtoxinA" as the non-proprietary established name for Allergan's specific formulation of botulinum toxin type A.
} 
A binds to the receptors in the presynaptic, cholinergic motor nerve terminal and is taken up by the nerve cells where the light chain of toxin cleaves a synaptosomeassociated protein (SNAP-25) to inhibit acetylcholine release from the nerve terminal. As its muscle relaxant effect is exerted in the hypertonic muscle, BoNTA offers an alternative treatment for spastic patients who have difficulty with oral muscle relaxants that can produce generalized weakness and drowsiness, cognitive impairment, and/or a reduced level of arousal. Locally injected BoNTA is expected to improve limb position and functional ability, and reduce pain in patients with spasticity. Moreover, BoNTA has no sedative action, unlike existing oral antispastic treatments, and therefore can be used in patients with cognitive impairment or a reduced level of arousal. Based on these considerations, BoNTA is a first-line treatment choice if the upper and lower limb spasticity is focal and reversible without contracture [2].

The efficacy and safety of BoNTA in patients with poststroke lower limb spasticity have been suggested by randomized-controlled trials of limited scale [3-8] and meta-analysis [9]. The efficacy of BoNTA in patients with severe brain injury has also been demonstrated in a randomized-control trial [10]. Approved treatments of spasticity in Japan include peripheral and central muscle relaxants, alcohol, phenol block, and intrathecal baclofen (only in cases of severe spastic paralysis). We conducted a clinical study to evaluate the efficacy and safety of BoNTA in Japanese patients with post-stroke lower limb spasticity who received a single placebo-controlled injection of BoNTA followed by open-label repeated treatment of up to three sessions. This article reports the efficacy and safety results of the double-blind phase.

\section{Methods}

A multicenter, randomized, double-blind, parallel-group, placebo-controlled study in Japanese patients with poststroke lower limb spasticity was conducted in 19 Japanese medical institutions in Japan between May 2007 and April 2008. The study was approved by the Institutional Review Board and was conducted according to the Declaration of Helsinki and the GCP; all patients signed informed consent.

\section{Patients}

Male or female patients aged 20-80 years and weighing at least $50 \mathrm{~kg}$ were eligible if they had had a stroke at least 6 months prior to treatment and had equinus deformity (plantar flexion of the ankle) as demonstrated by a score of $>3$ for ankle flexors on the Modified Ashworth Scale (MAS) [11].
Exclusion criteria were bilateral hemiplegia or quadriplegia; fixed contractures in the ankle; profound atrophy of the muscles to be injected; prior treatment with surgery, phenol/ethanol block, muscle afferent block (MAB), intrathecal baclofen, or any botulinum toxin serotype; and current use of peripheral muscle relaxants. Women were excluded if they were pregnant, lactating, potentially pregnant, or planning to become pregnant during the course of the study.

\section{Procedures}

The primary study objective was to confirm the superior efficacy of a single treatment of BoNTA $300 \mathrm{U}$ over placebo in patients with post-stroke lower limb spasticity using the MAS ankle score. MAS is widely used for the assessment of muscle tone and spasticity in lower limbs [5, 7-10]. Investigators were trained in the procedures to assess the MAS ankle score at the study start. Patients were assessed in the prone position, and the ankle was examined from the edge of the examination table for the MAS ankle score. The study included a screening examination (2-4 weeks before the treatment), a single treatment, and a 12-week follow-up period. Patients visited the center at screening, on the treatment day, and at weeks $1,4,6,8$, and 12 for specified examinations, observations, and assessments.

\section{Treatments}

\section{Investigational drug}

One vial of BoNTA (GSK1358820, BOTOX ${ }^{\circledR}$, Allergan, Inc., Irvine, CA) contains $100 \mathrm{U}$ of a specific formulation (Formulation 9060X) of botulinum toxin type A, $0.5 \mathrm{mg}$ human serum albumin, and $0.9 \mathrm{mg}$ sodium chloride per vial, which requires reconstitution prior to injection. The indistinguishable placebo contained $0.9 \mathrm{mg}$ sodium chloride per vial. Each vial was reconstituted with $8 \mathrm{ml}$ of non-preserved physiological saline resulting in a final concentration of $1.25 \mathrm{U}$ BoNTA per $0.1 \mathrm{ml}$ or placebo.

\section{Drug dose and muscle selection}

Drug dose and muscle selection was based on the Australian package insert [12]. Patients were randomly assigned to receive a single injection of $300 \mathrm{U}$ of BoNTA or placebo and were injected with $75 \mathrm{U}$ of BoNTA or placebo per muscle into each of the following: medial head of the gastrocnemius, lateral head of the gastrocnemius, and soleus muscle and tibialis posterior muscle (divided into three sites per muscle). Patients in the placebo group received the same volume of placebo solution into the same number of injections/muscles. 
An EMG or a nerve stimulator, and an EMG injection needle were used to identify the proper muscles and facilitate injection in all patients.

\section{Randomization}

After screening, each eligible patient was randomized 1:1 to receive either BoNTA or placebo injection on the treatment day. The person responsible for randomization prepared a randomization code table, and it was concealed from all investigators and all study personnel until data collection and analysis were completed.

Assessments

\section{Efficacy}

MAS The muscle tone of the ankle was assessed by the investigator using the MAS [11] at screening, pre-injection on the treatment day (baseline), and at weeks 1, 4, 6, 8, and 12 or at study withdrawal.

Gait pattern scale (physician's rating scale) The investigator assessed the gait pattern while the patient walked $10 \mathrm{~m}$ using the Physician's Rating Scale [13] (Table 1) pre-injection on the treatment day and at weeks $1,4,6,8$, and 12 or at study withdrawal. This scale of -1 (worst) to 9 (best) based on three parameters (initial foot contact, foot

Table 1 Gait pattern scale (Physician's Rating Scale)

\begin{tabular}{llc}
\hline Gait parameter & Definition & $\begin{array}{c}\text { Limb } \\
\text { score }\end{array}$ \\
\hline Initial foot contact & Toe & 0 \\
& Forefoot & 1 \\
& Foot-flat & 2 \\
& Heel & 3 \\
Foot contact at & Toe/toe (equinus) & -1 \\
midstance & Foot-flat/early heel rise & 0 \\
& Foot-flat/no early heel rise & 1 \\
& Occasional heel/foot-flat & 2 \\
Gait assistive & Heel/toe (normal roll-over) & 3 \\
devices & Walker (forward/posterior) with & 0 \\
& assistance & \\
& Walker (independent) & 2 \\
Total score (score $=$ & Crutches, sticks & 3 \\
\hline
\end{tabular}

Adapted from: Boyd RN, Graham HK. Objective measurement of clinical findings in the use of botulinum toxin type A for the management of children with cerebral palsy. European Journal of Neurology. 1999;(4):S23-S35 (Partial quoted) contact at midstance, and gait-assisting devices) is an observational gait scale originally developed by Koman et al. [14]., modified by Corry [15], and subsequently modified by Boyd et al. [13]. In this study, gait parameters that were suitable for the intended purpose were selected from the original parameters.

Speed of gait The investigator or clinical research coordinator measured the time (seconds) the patient took to walk $10 \mathrm{~m}$ straight ahead pre-injection on the treatment day and at weeks $1,4,6,8$, and 12 or at study withdrawal.

Clinical global impression (CGI) At screening, preinjection on the treatment day, and at weeks $1,4,6$, 8, and 12 or at study withdrawal, the global impression of functional disability on the visit day was assessed using the Numeric Rating Scale, with -5 indicating worst possible and 5 best possible rating, by the investigator, the patient, and the physical or occupational therapist.

Safety Adverse events were recorded throughout the study. Laboratory tests were performed at screening and at week 12 or at study withdrawal. Vital sign assessments (pulse rate and blood pressure) and 12-lead electrocardiography were performed on the treatment day and week 12 or at study withdrawal.

\section{Data analysis}

The sample size was chosen based on the previous study [6]. Assuming a mean difference of 5 points in the AUC for the MAS ankle score change between BoNTA and placebo based on the results and a SD value of 7.5 for both treatments, 49 subjects per group were be required to provide $90 \%$ power at the $5 \%$ level of significance (two-sided) using t-test to achieve superiority over placebo. Assuming approximately $20 \%$ of dropouts, the sample size of 60 subjects per group (120 subjects in total) was chosen.

Efficacy data were analyzed using the full analysis set (FAS), defined as all randomized patients that received study treatment and had at least one MAS ankle assessment after treatment.

The AUC of the change from baseline in the MAS ankle score was the primary endpoint. Considering the differences of individual peak efficacy, the assessment over the treatment period by AUC as summary index was considered to provide more accurate evaluation than assessment at a specific time point [16-18], resulting in more accurate evaluation of efficacy of BoNTA compared to placebo. MAS ankle scores at individual time points were also evaluated. For each patient, the change from baseline (the day of the treatment) in the MAS ankle score at each time point was calculated; " $1+$ " was analyzed as score 1.5. 
Fig. 1 Study patient flow chart. BoNTA Botulinum toxin type A

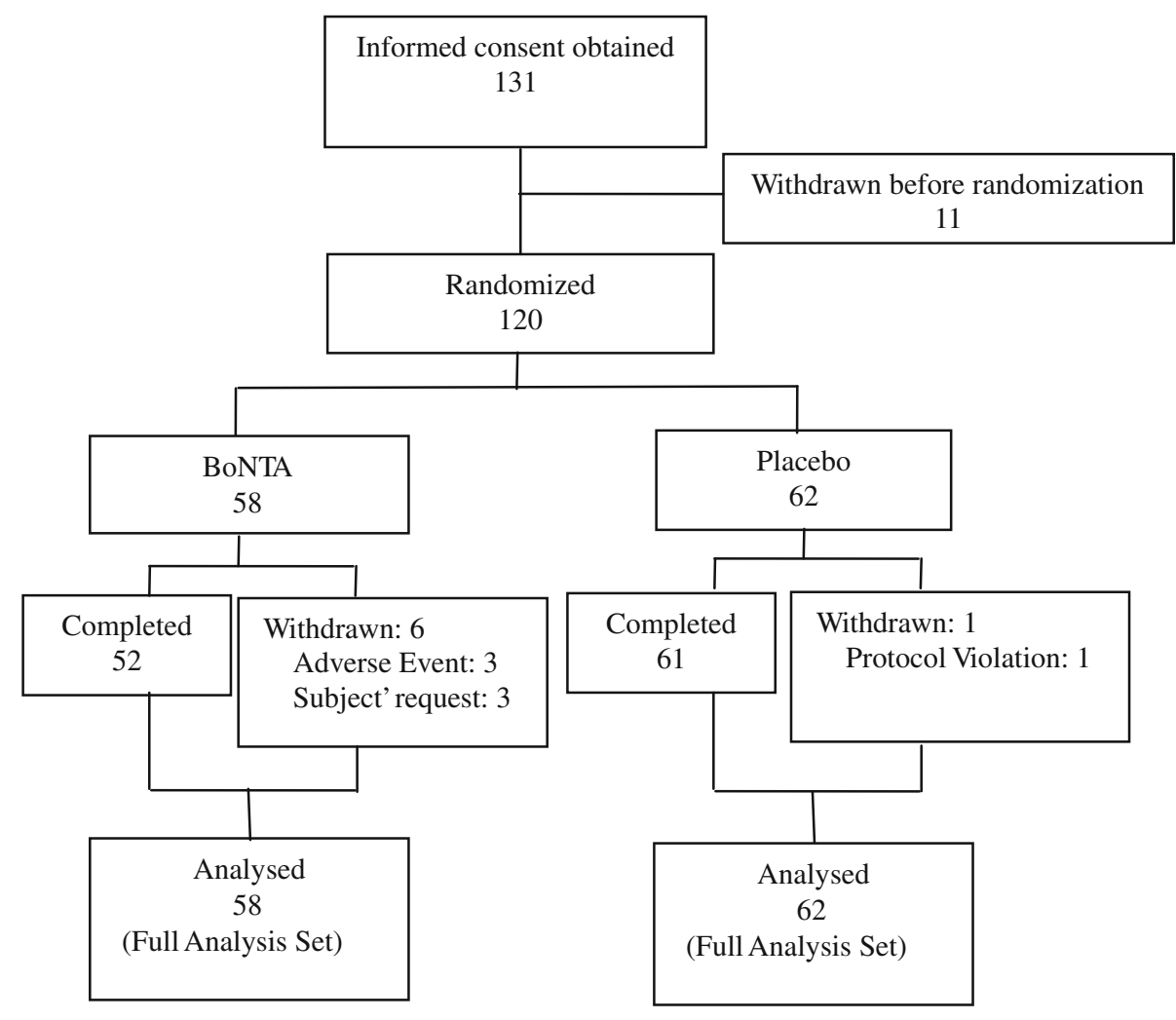

BoNTA=Botulinum toxin type $\mathrm{A}$
Changes from baseline (vertical axis) were plotted against time (horizontal axis), and the area under the resultant curve was obtained. A decrease from baseline resulted in a negative (-) AUC value, while an increase from baseline resulted in a positive (+) AUC value. The difference in the mean AUC between the BoNTA group and the placebo group was tested using a $t$-test. The point estimate and the 95\% CI of the mean group difference were also calculated.

Changes from baseline in the MAS ankle score, the Physician's Rating Scale, the speed of gait, and the CGI at each time point were summarized by group; the Wilcoxon test was used to determine statistical differences between the BoNTA group and the placebo group.

Safety data were analyzed for the safety population (SP), defined as all patients that received study treatment.

\section{Results}

Demographics

Of the 131 patients who provided informed consent, 120 were randomized and received study treatment (58 BoNTA, 62 placebo). One hundred thirteen patients completed the study (Fig. 1). Seven patients prematurely withdrew after randomization: three (BoNTA group) due to adverse events, three (BoNTA group) consent withdrawal, and one (placebo group) deviation from the protocol (met exclusion criteria). Demographic and baseline characteristics except gender were comparable between the groups. More men than women were included in both groups (Table 2).

Primary endpoint

AUC of the change from baseline in MAS ankle scores

After treatment the ankle MAS AUC was significantly lower in the BoNTA group than in the placebo groups, demonstrating the superiority of BoNTA to placebo (mean difference, $-3.428 ; 95 \% \mathrm{CI},-5.841$ to -1.016 ; $p=0.006$; $t$ test) (Fig. 2).

Secondary endpoints

$M A S$

After treatment, in the ankle MAS at individual time points a significantly greater decrease from baseline was noted at weeks 4,6 , and 8 in the BoNTA group compared to the placebo group $(p<0.001)$ (Fig. 3; Table 3). 
Table 2 Demographics and patient characteristics (FAS population)

\begin{tabular}{lll}
\hline & BoNTA & Placebo \\
& $N=58$ & $N=62$ \\
\hline Age (years) & $62.4 \pm 8.7$ & $62.5 \pm 9.3$ \\
Race (Japanese) & 58 & 62 \\
Gender (female/male) & $8 / 50$ & $16 / 46$ \\
Weight (kg) & $67.6 \pm 10.0$ & $65.0 \pm 9.5$ \\
Mean duration of stroke (month) & $80.8 \pm 72.8$ & $72.0 \pm 60.3$ \\
MAS & $3.28 \pm 0.45$ & $3.24 \pm 0.43$ \\
Gait pattern scale (Physician's & $3.10 \pm 2.35$ & $3.23 \pm 2.02$ \\
$\quad$ Rating Scale) & & \\
Speed of gait (s) & & \\
CGI & $60.96 \pm 49.69$ & $52.83 \pm 51.57$ \\
Investigator & $-1.33 \pm 1.69$ & $-1.26 \pm 1.78$ \\
Patient & $-1.28 \pm 1.89$ & $-0.92 \pm 2.05$ \\
Physical/occupational therapist & $-1.26 \pm 2.00$ & $-1.23 \pm 2.04$ \\
\hline
\end{tabular}

Values are mean $\pm \mathrm{SD}$, except where stated otherwise

a Time (s) that the patients took to walk $10 \mathrm{~m}$ straight ahead

BoNTA Botulinum toxin type A, CGI clinical global impression, FAS full analysis set, MAS Modified Ashworth Scale

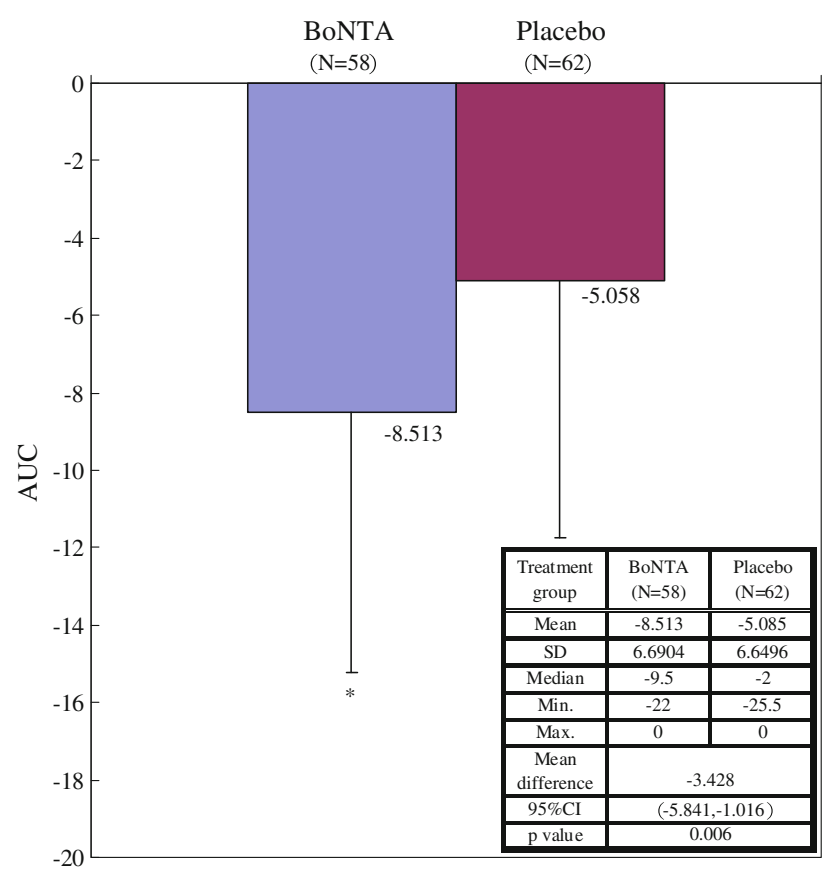

Fig. 2 Mean AUC for MAS of ankle. AUC area under the curve, BoNTA Botulinum toxin type A, MAS Modified Ashworth Scale. $* p<0.05$ ( $t$ test). AUC was obtained by plotting changes from baseline (vertical axis) of MAS against time point (horizontal axis). The "1+" was analyzed as score 1.5. A decrease from baseline resulted in a negative (-) AUC value

\section{Gait scale (physician's rating scale)}

After treatment a slight increase from baseline in the Physician's Rating Scale score was noted for both groups, and no significant group difference was observed at any time point (Table 3 ).

\section{Gait speed}

Gait speed measured as the time required for a $10-\mathrm{m}$ walk started to decrease at week 4 in the BoNTA group; however, the same trend was noted in the placebo group, and no significant group difference was observed at any time point (Table 3).

\section{$C G I$}

There was a significantly greater increase in the CGI score by the investigator noted in the BoNTA group compared to the increase in the CGI in the placebo group at weeks 4,6 , and 8 ( $p=0.016$ to 0.048 , Wilcoxon test).

No significant group difference was noted in the CGI scores by the patient and by the physical or occupational therapist at any time point (Table 3).

\section{Safety}

\section{Adverse events}

Adverse events were reported in $45 \%$ (26/58) in the BoNTA group and $44 \%(27 / 62)$ in the placebo group during the 12-week follow-up period.

Serious adverse events were reported in $9 \%(5 / 58)$ in the BoNTA group and 2\% (1/62) in the placebo group during the 12-week follow-up period. All of the serious adverse events except for myalgia were considered to be unrelated to BoNTA. All of these events were resolved.

Investigator-determined treatment-related adverse events were reported during the 12-week follow-up period in $12 \%(7 / 58)$ in the BoNTA group and $11 \%(7 / 62)$ in the placebo group (Table 4). All of the treatment-related adverse events reported in the BoNTA group were mild or moderate in intensity.

\section{Other safety endpoints}

There were no marked changes in any laboratory data, blood pressures, pulse rates, or EKGs in any group after the treatment.

\section{Discussion}

This study demonstrated the superiority of $300 \mathrm{U}$ BoNTA to placebo to reduce muscle tone based on the AUC of the change from baseline in the MAS ankle score as the primary efficacy endpoint. At individual time points the MAS 


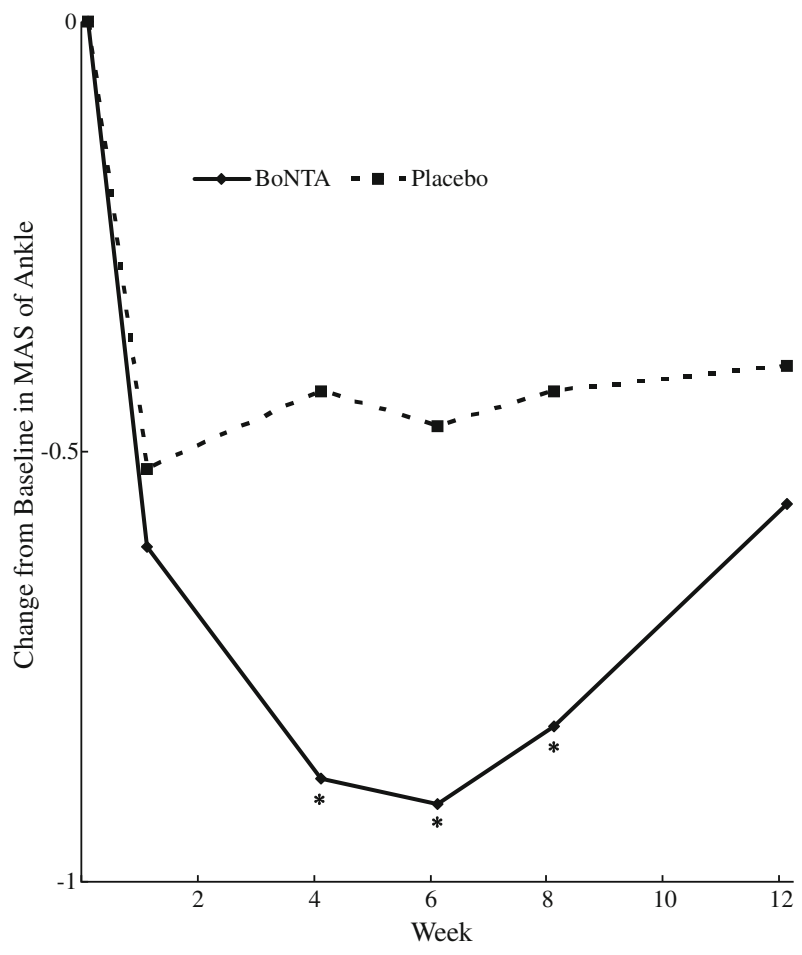

Fig. 3 Mean of change from baseline in MAS of ankle. BoNTA Botulinum toxin type A, MAS Modified Ashworth Scale. ${ }^{*} p<0.05$ (Wilcoxon test)

ankle score showed a significantly greater decrease from baseline for the BoNTA group compared to placebo at 4, 6 , and 8 weeks after treatment. These results demonstrated the efficacy of BoNTA injections for improvement of spasticity in the ankle in Japanese patients by the same action mechanism of BoNTA as in Caucasian patients demonstrated in previous studies [3-10].

Patients with spasticity can suffer from impaired walking ability that includes equinus foot; consequently this disability negatively affects performance of the patient's activities of daily living (ADL). As secondary endpoints the patient's gait pattern and gait speed were assessed to determine the efficacy of BoNTA for improving these measures of ambulation. Both BoNTA and placebo treatments were associated with slight increases in the Physician's Rating Scale score (i.e., gait pattern scale), and no significant difference was noted between BoNTA and placebo. In addition, the time required for 10-m walking decreased after treatment with either BoNTA or placebo, and no significant difference was noted between the two treatments.

The lack of effect on ambulation for subjects treated with BoNTA may be due to their need to change from spastic gait attributable to talipes equinovarus to the gait with normal grounding of the foot through alleviation of the spasticity of the ankle. Furthermore, the mean duration of stroke in this study was 76.3 months (approximately
Table 3 Mean and SD of change from baseline in MAS, PRS, speed of gait, and CGI

\begin{tabular}{|c|c|c|c|c|c|}
\hline & \multicolumn{2}{|c|}{ BoNTA $(N=58)$} & \multicolumn{2}{|c|}{ Placebo $(N=62)$} & \multirow[t]{2}{*}{$p$ value $^{\mathrm{a}}$} \\
\hline & $n$ & Mean \pm SD & $n$ & Mean \pm SD & \\
\hline \multicolumn{6}{|l|}{ MAS } \\
\hline Week 4 & 56 & $-0.88 \pm 0.69$ & 62 & $-0.43 \pm 0.72$ & $<0.001$ \\
\hline Week 8 & 54 & $-0.82 \pm 0.66$ & 61 & $-0.43 \pm 0.68$ & $<0.001$ \\
\hline Week 12 & 54 & $-0.56 \pm 0.69$ & 61 & $-0.40 \pm 0.58$ & 0.240 \\
\hline \multicolumn{6}{|c|}{ Gait pattern scale (Physician's Rating Scale) } \\
\hline Week 4 & 54 & $0.54 \pm 1.22$ & 60 & $0.63 \pm 1.45$ & 0.688 \\
\hline Week 8 & 54 & $0.61 \pm 1.19$ & 59 & $0.78 \pm 1.81$ & 0.825 \\
\hline Week 12 & 53 & $0.55 \pm 1.26$ & 60 & $0.58 \pm 1.57$ & 0.775 \\
\hline \multicolumn{6}{|l|}{ Speed of gait } \\
\hline Week 4 & 54 & $-6.10 \pm 22.86$ & 61 & $-7.37 \pm 20.78$ & 0.209 \\
\hline Week 8 & 54 & $-3.14 \pm 35.78$ & 60 & $-8.19 \pm 18.63$ & 0.367 \\
\hline Week 12 & 53 & $-10.14 \pm 26.93$ & 61 & $-8.53 \pm 24.71$ & 0.585 \\
\hline \multicolumn{6}{|l|}{ CGI } \\
\hline \multicolumn{6}{|l|}{ Investigator } \\
\hline Week 4 & 56 & $1.09 \pm 1.25$ & 61 & $0.64 \pm 1.07$ & 0.048 \\
\hline Week 8 & 54 & $1.13 \pm 1.32$ & 61 & $0.59 \pm 1.23$ & 0.016 \\
\hline Week 12 & 53 & $0.81 \pm 1.30$ & 61 & $0.52 \pm 1.27$ & 0.166 \\
\hline \multicolumn{6}{|l|}{ Patient } \\
\hline Week 4 & 56 & $0.75 \pm 1.58$ & 61 & $0.44 \pm 1.76$ & 0.120 \\
\hline Week 8 & 54 & $1.00 \pm 2.03$ & 61 & $0.70 \pm 1.98$ & 0.281 \\
\hline Week 12 & 53 & $0.49 \pm 1.53$ & 61 & $0.49 \pm 2.18$ & 0.409 \\
\hline \multicolumn{6}{|c|}{ Physical/occupational therapist } \\
\hline Week 4 & 54 & $0.64 \pm 1.27$ & 61 & $1.07 \pm 1.38$ & 0.119 \\
\hline Week 8 & 53 & $1.04 \pm 1.78$ & 61 & $1.00 \pm 1.64$ & 0.589 \\
\hline Week 12 & 53 & $1.02 \pm 1.34$ & 61 & $0.97 \pm 1.51$ & 0.600 \\
\hline
\end{tabular}

a Wilcoxon test

BoNTA Botulinum toxin type A, CGI clinical global impression, MAS Modified Ashworth Scale, PRS Physician's Rating Scale

6 years). This extended duration of spasticity would allow for accommodation of physical changes as patients become accustomed to walk making use of their spasticity, as set motor patterns are changed with rearrangement of underlying neural circuits. This short-duration study (3 months) may be insufficient to learn the gait with normal grounding of the foot and adequately modify the neural circuit. Therefore, the patients' gait scale was not improved much.

It may be possible that rehabilitation could be facilitated after treatment with BoNTA, but that assessment is beyond the scope of this study. In this study patients were allowed concomitant use of rehabilitation, "but the frequency and content of rehabilitation should not be changed" during this study. Since the rehabilitation training was not designed or modified to accommodate for the change in muscle tone associated the synergic effect of rehabilitation including the walk training and BoNTA administration did not meet expectations. The effects of BoNTA on gait 
Table 4 Summary of drug-related adverse events in the 12-week double-blind phase

\begin{tabular}{lll}
\hline $\begin{array}{l}\text { Drug-related adverse events/ } \\
\text { preferred term }\end{array}$ & $\begin{array}{l}\text { BoNTA } \\
(N=58)\end{array}$ & $\begin{array}{l}\text { Placebo } \\
(N=62)\end{array}$ \\
\hline Any event $(\%)^{\mathrm{a}}$ & $7(12 \%)$ & $7(11 \%)$ \\
Myalgia & $3(5 \%)$ & $2(3 \%)$ \\
Injection site pain & $3(5 \%)$ & $1(2 \%)$ \\
Administration site pain & $1(2 \%)$ & 0 \\
Joint sprain & $1(2 \%)$ & 0 \\
Erythema & 0 & $1(2 \%)$ \\
Gamma-glutamyltransferase increased & 0 & $1(2 \%)$ \\
Pain in extremity & 0 & $1(2 \%)$ \\
Sinus tachycardia & 0 & $1(2 \%)$ \\
\hline
\end{tabular}

${ }^{a}$ One patient may have two or more events

BoNTA Botulinum toxin type A

pattern determined by the Physician's Rating Scale and gait speed should be further assessed in a long-term treatment setting where learning a modified motor pattern and the underlying neural modifications may be established. Possible improvement in equinus foot after long-term BoNTA treatment with combined use of rehabilitation is expected to allow correction of walking patterns and thereby reduce the systemic burden of walking. Increasing walking distance (or decreased time required for a given walk) may expand the range of patient activities and lead to improved ADL. Further long-term study in the open label extension is important to clarify the perceived benefits and changes in the ADL and quality of life.

A significantly greater increase in the CGI score by the investigator was noted in the BoNTA group compared to the placebo group at weeks 4,6 , and 8 , but no significant difference was noted by the patient or physical/occupational therapist. Since the result of the Physician's Rating Scale score and speed of gait was not significant in this study, the treatment outcome seems to have fallen short of the expectation of patients and the physical/occupational therapist. These results differ from previous reports $[9,19]$ that BoNTA improves the impression of functional disability assessed by the Global Assessment Scale. Beneficial effects of BoNTA on gait pattern and speed of gait may be more apparent after a longer treatment period.

No marked difference was noted in the frequency of treatment-related adverse events between groups [7 (12\%) with BoNTA vs. $7(11 \%)$ with placebo]. Injection or administration site pain was reported in four patients (7\%) in the BoNTA group and one patient $(2 \%)$ in the placebo group. This is an event associated with intramuscular injection, and caution should be exercised in injecting BoNTA. There were no reports of treatment-related adverse events characteristic of oral muscle relaxants (sleepiness, feelings of weakness, and a light-headed feeling). In this study, the safety of BoNTA was similar to observations from a pooled analysis of the safety of the same formulation of BoNTA that included nine doubleblind, placebo-controlled studies of spasticity patients after stroke [20].

An important consideration for the use of BoNTA is the serotype and formulation. As BoNTA is a biological product, it is not interchangeable with other products because of the complexity, between-product variability, and specificity of the manufacturing processes. Consequently, botulinum toxin products are non-interchangeable with any other botulinum toxins [21-27].

The results of this study indicate the efficacy of BoNTA $300 \mathrm{U}$ without any dose-limiting adverse events. This study demonstrated the safety and efficacy of BoNTA $300 \mathrm{U}$ into the muscles of lower limbs of patients with post-stroke ankle spasticity.

As a drug product for local administration, BoNTA can be directly injected into a target muscle with localized hypertonia to relax the muscle. Its direct relaxant effect on the muscle causing spasticity may reduce equinus foot and improve walking ability, leading to the improved ADL performance of patients. This effect is expected to reduce the dependence on care when walking inside or outside as well as to reduce the burden for patients, family, and caregivers. The observed persistence of the efficacy of a single BoNTA treatment for 12 weeks suggests possible better treatment compliance compared to the use of oral muscle relaxants that require daily medication [28]. Moreover, BoNTA may be an effective treatment choice for patients who have difficulty treating spasticity with oral muscle relaxants because of adverse drug reactions.

The long-term efficacy and safety, and the effects on rehabilitation of BoNTA will be evaluated using the data obtained in the open-label phase.

Acknowledgment The authors thank the investigators of GSK1358820 Spasticity Study Group who enrolled their patients in this study: Masahiro Abo, The Jikei University School of Medicine, Tokyo; Haruki Chagawa, Iwakuni Medical Center, Yamaguchi; Kouzou Fukuyama, Fukuoka Wajiro Hospital, Fukuoka; Yasumi Igarashi, Aida Kinen Rehabilitation Hospital, Ibaraki; Haruo Inada, Nakaizu Rehabilitation Center, Shizuoka; Kou Oka, Oka Neurocare Clinic, Shizuoka; Takashi Nagayama, Tsurumaki Onsen Hospital, Kanagawa; Hijiri Ito, Mifukai Vihara Hananosato Hospital, Hiroshima; Tetsumasa Kamei, Chigasaki Tokushukai Medical Center, Kanagawa; Fumihito Kasai, Showa University Fujigaoka Rehabilitation Hospital, Kanagawa; Junya Kawada, Shonan Kamakura General Hospital, Kanagawa; Nobuyuki Kawate, Showa University Hospital, Tokyo; Akio Kimura, Keio University Tsukigase Rehabilitation Center, Shizuoka; Kaoru Matsunaga, Kumamoto Kinoh Hospital, Kumamoto; Masazumi Mizuma, Showa University Hospital, Tokyo; Toshikazu Ogasawara, Minami-Sapporo Neurosurgical Clinic, Hokkaido; Shinzo Oota, Brain Attack Center Oota Memorial 
Hospital, Hiroshima; Tadakazu Shimizu, Sapporo Shuyukai Hospital, Hokkaido; Rihee Takeda, Nakamura Memorial South Hospital, Hokkaido; Shigeki Yura, Tomakomai Nissho Hospital, Hokkaido. The authors also thank all the participating patients for their time and efforts. This protocol was posted on the Clinicaltrials.gov database, identifier \#NCT00460655.

Conflict of interest statement This study was sponsored by GlaxoSmithKline K.K. Dr. Kaji served on the steering committee of GSK1358820 Spasticity Study and received grants from GlaxoSmithKline K.K. He also receives honoraria for speaker's bureau activities from Eisai Co., Ltd. Yuka Osako, Kazuaki Suyama, Toshio Maeda, Dr. Uechi, and Dr. Iwasaki are employed by GlaxoSmithKline K.K.

Open Access This article is distributed under the terms of the Creative Commons Attribution Noncommercial License which permits any noncommercial use, distribution, and reproduction in any medium, provided the original author(s) and source are credited.

\section{References}

1. Lance JW (1980) Symposium synopsis. In: Feldman RG, Young RR, Koella WP (eds) Spasticity: disordered motor control. Year Book Medical Publishers, Chicago, pp 485-494

2. Ward AB (2002) A summary of spasticity management-a treatment algorithm. Eur J Neurol 9(Suppl 1):48-52

3. Grazko MA, Polo KB, Jabbari B (1995) Botulinum toxin A for spasticity, muscle spasms and rigidity. Neurology 45:712-717

4. Burbaud P, Wiart L, Dubos JL et al (1996) A randomised, double blind, placebo controlled trial of botulinum toxin in the treatment of spastic foot in hemiparetic patients. J Neurol Neurosurg Psychiatry 61:265-269

5. Reiter F, Danni M, Lagalla G, Ceravolo G, Provinciali L (1998) Low-dose botulinum toxin with ankle taping for the treatment of spastic equinovarus foot after stroke. Arch Phys Med Rehabil 79:532-535

6. Dunne J (2002) Botulinum toxin type A (BOTOX) in the treatment of lower limb spasticity during stroke rehabilitation. Intern Med J 33(Suppl):A41

7. Mancini F, Sandrini G, Moglia A, Nappi G, Pacchetti C (2005) A randomised, double-blind, dose-ranging study to evaluate efficacy and safety of three doses of botulinum toxin type A (Botox) for the treatment of spastic foot. Neurol Sci 26:26-31

8. Farina S, Migliorini C, Gandolfi M et al (2008) Combined effects of botulinum toxin and casting treatments on lower limb spasticity after stroke. Funct Neurol 23(2):87-91

9. Rosales RL, Chua-Yap AS (2008) Evidence-based systematic review on the efficacy and safety of botulinum toxin-A therapy in post-stroke spasticity. J Neural Transm 115:617-623

10. Verplancke D, Snape S, Salisbury CF, Jones PW, Ward AB (2005) A randomized controlled trial of botulinum toxin on lower limb spasticity following acute acquired severe brain injury. Clin Rehabil 19:117-125

11. Bohannon RW, Smith MB (1987) Interrater reliability of a Modified Ashworth Scale of muscle spasticity. Phys Ther 67:206-207

12. BOTOX ${ }^{\circledR}$ Botulinum Toxin Product Information Version 1.0 (2008) Allergan Australia Pty. Limited

13. Boyd RN, Graham HK (1999) Objective measurement of clinical findings in the use of botulinum toxin type A for the management of children with cerebral palsy. Eur J Neurol 6(suppl 4):S23-S35

14. Koman LA, Mooney JF III, Smith BP, Goodman A (1993) Management of cerebral palsy with botulinum toxin A: preliminary investigation. J Pediatr Orthop 13:489-495

15. Corry IS, Cosgrove AP, Duffy CM, McNeill S, Eames N, Taylor TC, Graham HK (1998) Botulinum toxin A compared with stretching casts in the treatment of spastic equinus: a randomised prospective trial. J Paediatr Orthop 18:304-311

16. Matthews JNS, Altman DG, Campbell MJ, Roytson P (1990) Analysis of serial measurements in medical research. BMJ 300:230-235

17. Liu C, Li Wan Po A, Blumhardt LD (1998) "Summary measure" statistic for assessing the outcome of treatment trials in relapsingremitting multiple sclerosis. J Neurol Neurosurg Psychiatry 64:726-729

18. Pham B, Cranney A, Boers M, Verhoeven AC, Wells G, Tugwell $P$ (1999) Validity of area-under-the-curve analysis to summarize effect in rheumatoid arthritis clinical trials. J Rheumatol 26(3):712-716

19. Smith SJ, Ellis E, White S, Moore AP (2000) A double-blind placebo-controlled study of botulinum toxin in upper limb spasticity after stroke or head injury. Clin Rehabil 14:5-13

20. Turkel CC, Bowen B, Liu J, Brin MF (2006) Pooled analysis of the safety of botulinum toxin type A in the treatment of poststroke spasticity. Arch Phys Med Rehabil 87:786-792

21. Anderson TJ, Rivest J, Stell R et al (1992) Botulinum toxin treatment of spasmodic torticollis. J R Soc Med 85:524-529

22. Brin MF, Blitzer A (1993) Botulinum toxin: dangerous terminology errors. J R Soc Med 86:493-494

23. Marsden CD (1993) Botulinum toxin: dangerous terminology errors-reply. J R Soc Med 86:494

24. Chapman MA, Barron R, Tanis DC, Gill CE, Charles PD (2007) Comparison of botulinum neurotoxin preparations for the treatment of cervical dystonia. Clin Ther 29:1325-1337

25. Wenzel R, Jones D, Borrego JA (2007) Comparing two botulinum toxin type A formulations using manufacturers' product summaries. J Clin Pharm Ther 32:387-402

26. Harper L (2009) Botulinum toxin A-when is a unit not a unit? J Urol 181:414-415

27. BOTOX ${ }^{\circledR}$ Product Information (2009) Allergan, Inc

28. Simpson DM, Gracies JM, Yablon SA, Barbano R, Brashear A (2009) Botulinum neurotoxin versus tizanidine in upper limb spasticity: a placebo-controlled study. J Neurol Neurosurg Psychiatry 80:380-385 